\title{
Sichere Fixierung hydrophiler Gleitdrähte mittels Klemmchen
}

\author{
Bei der Ureterorenoskopie wird häufig ein ultraflexibler, hydrophiler Gleitdraht verwendet. \\ Dieser kann jedoch beim Entfernen des Ureterkatheters leicht verschoben werden. \\ Unsere Autoren empfehlen deashalb eine Fixierung mit OP-Klemmchen.
}

Endoskopische Eingriffe zur Therapie einer Urolithiasis oder bei ureteralen Harntransportstörungen gehören zum urologischen Alltag. Nach retrograder Darstellung kommt bei der (flexiblen) Ureterorenoskopie (URS) häufig ein ultraflexibler, hydrophiler Gleitdraht (Teru$\mathrm{mo}^{\circledR}$ ) als gewebeschonende Führungshilfe zum Einsatz. Nach korrekter Platzierung des Drahtes im Nierenbecken durch den Ureterkatheter (UK) wird dieser über den Führungsdraht wieder entfernt. In unserer Klinik erfolgt anschließend routinemäßig eine Ostien/Harnleiterbougierung über den einliegenden Draht. Die Technik geht auf den schwedischen Radiologen Sven-Ivar Seldinger zurück [1]. Nachfolgend belassen wir den Draht und führen das Ureterorenoskop parallel dazu in den Ureter ein. Damit bleibt in jedem Fall eine sichere Schienung des Harnleiters gewährt und der Arbeitskanal des Instruments z. B. für Fasszangen passierbar.
Beim Entfernen des UK oder anderer Instrumente über den Draht (oder auch parallel davon) kann es aufgrund der extrem flexiblen und hydrophilen Struktur leicht zu einer Dislokation des Drahtes kommen. Dies ist mit längerer OP-Dauer, höheren Durchleuchtungszeiten und steigendem Komplikationsrisiko für den Patienten verbunden.

Ein einfacher und schneller Trick zur Vermeidung einer Dislokation des hydrophilen Gleitdrahtes, die mit nassen Handschuhen oft nicht bemerkt wird, ist die Fixierung des Drahtes mittels eines kleinen OP-Klemmchens. Bei jedem Zurückziehen von UK oder DJ-Harnleiterschiene wird der Draht durch den Operateur mittels Klemmchen fixiert (○ Abb.1). Diese Klemmchen-Fixierung führt zudem bei einem Terumo $^{\circledR}$-Draht (anders als bei Teflon ${ }^{\circledR}$-Drähten) nicht zu einer Quetschung/Entrundung, wodurch ein an- schließendes Vorschieben von Harnleiterschienen weiter möglich ist.

\section{Dr. Stephan Degener,}

Prof. Dr. Stephan Roth

\section{Literatur}

1 Seldinger SI. Catheter replacement of the needle in percutaneous arteriography; a new technique. Acta radiol 1953; 39: 368 376

\section{Korrespondenz:}

Dr. Stephan Degener

Klinik für Urologie und Kinderurologie

Zentrum für Forschung in der klinischen

Medizin (ZFKM)

Universität Witten/Herdecke

Helios Klinikum Wuppertal

Heusnerstraße 40

42283 Wuppertal

stephan.degener@helios-kliniken.de
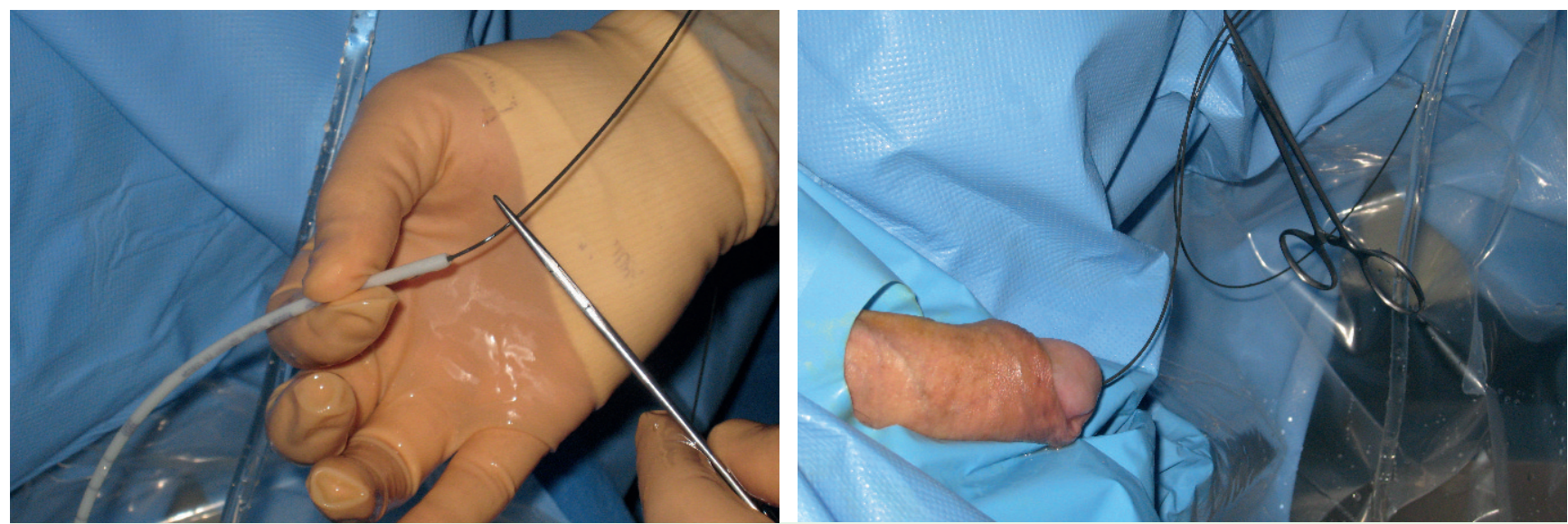

Abb.1 Ein steril abgedeckter Patient in Steinschnittlage bei einer URS. Der hydrophile Gleitdraht wird mittels kleinem OP-Klemmchen fixiert - durch den Operateur im Rahmen der Harnleiterbougierung (links) oder am Abdecktuch für die Ureterorenoskopie (rechts). 